\title{
A Quantum Biological Switch Based on Superradiance Transitions
}

\author{
D. Ferrari,${ }^{1}$ G.L. Celardo, ${ }^{1,2}$ G.P. Berman, ${ }^{3}$ R.T. Sayre,${ }^{4}$ and F. Borgonovi ${ }^{1,2}$ \\ ${ }^{1}$ Dipartimento di Matematica e Fisica Università Cattolica, \\ via Musei 41, 25121 Brescia, Italy \\ 2 Interdisciplinary Laboratories for Advanced Materials Physics, \\ via Musei 41, 25121 Brescia, Italy \\ Istituto Nazionale di Fisica Nucleare, \\ Sezione di Pavia, via Bassi 6, I-27100, Pavia, Italy \\ ${ }^{3}$ Theoretical Division, MS-B213, Los Alamos National Laboratories, Los Alamos, NM USA, \\ 4 Los Alamos National Laboratory and New Mexico Consortium, \\ 202B Research Center, Los Alamos, NM 87544, USA
}

(Dated: February 28, 2022)

\begin{abstract}
A linear chain of connected electron sites with two asymmetric sinks, one attached to each end, is used as a simple model of quantum electron transfer in photosynthetic bio-complexes. For a symmetric initial population in the middle of the chain, it is expected that electron transfer is mainly directed towards the strongest coupled sink. However, we show that quantum effects radically change this intuitive "classical" mechanism, so that electron transfer can occur through the weaker coupled sink with maximal efficiency. Using this capability, we show how to design a quantum switch that can transfer an electron to the left or right branch of the chain, by changing the coupling to the sinks. The operational principles of this quantum device can be understood in terms of superradiance transitions and subradiant states. This switching, being a pure quantum effect, can be used as a witness of wave-like behaviour of excitations in molecular chains. When realistic data are used for the photosystem II reaction center, this quantum biological switch is shown to retain its reliability, even at room temperature.
\end{abstract}

PACS numbers: 05.60.Gg, 03.65.Yz, 72.15.Rn 


\section{INTRODUCTION.}

Understanding how biological systems transfer and store energy is a basic energy science challenge that can lead the design of new bio-nanotechnological devices [1 5].

Recent experiments on photosynthesis by several groups [6-14], have demonstrated the striking role of quantum coherence in the form of long lasting oscillations of the population of excitonic states in light harvesting complexes (LHC), at room temperature. Even though there is no general consensus on the role quantum coherence plays in the electron transfer (ET) efficiency $(\simeq 99 \%)[15-20$, there is no doubt that the models for exciton transport in the LHCs and primary charge separation in the reaction centers (RCs) should utilize quantum coherent effects.

The photosystem II (PSII) RC of many bacteria, plants and algae, where the primary charge separation occurs, is arranged in two symmetric branches, even if only one of them is active for the ET. Different mechanisms which could be responsible for the asymmetry in the ET in the PSII RCs, and the related experiments, are discussed in [21 30] (see also references therein).

Here we do not address the question why only one branch is active, but we use the PSII $\mathrm{RC}$ as a prototype for an artificial biological switch, able to drive the ET to the left or the right symmetric branch, by controlling the couplings to the sinks.

Primary charge separation in the $\mathrm{RC}$ can be modeled starting from a donor (a dimer, called the special pair where the excitation starts) and then including the ET through different protein subunits, (bacterio)chlorophylls and (bacterio)pheophytins, generally called chromophores. This transfer occurs in a very short time (a few picoseconds). On the other hand, the effective ET to the quinone occurs over a much longer time (a few microseconds) [26 33]. This allows one to consider a simplified model for the RC taking into account the short time dynamics corresponding to primary charge separation (disregarding longtime effects) by adding sinks through which the electron can escape the system. While there exists much experimental data for calculating both the energy levels and the couplings between the chromophores (typically dipole-dipole or Coulomb interactions), our aim here

is to avoid non-essential technicalities which complicate the model, and concentrate on the main ideas of designing a quantum switching device based on the ET in the PSII RC.

The model can be described within the framework of an effective non-Hermitian Hamil- 
tonian which takes into account the loss of electron probability to the sinks, one attached to each end of the system. Even though this scheme has been used in the past [26, 27, 34, 36], to the best of our knowledge the intrinsic mechanism of superradiance and its relation to the ET has not been fully understood in bio-systems. In bio-system superradiance has been considered as an effect of the coupling with the electromagnetic field [37] but actually it is a generic effect [38] which can occur when a system is coupled to any continuum of states. Here we consider the phenomenon of superradiance in transport induced by the coupling with the continuum of scattering states as been discussed in 34. This point of view is related to the supertransfer phenomenon discussed in Ref. [39].

\section{THE MODEL.}

The model we consider consists of six sites divided into two symmetric branches, left and right, with two independent sinks attached at the ends. For simplicity, the energies of the sites are taken to be equal, $E_{0}=0$, and the coupling between the nearest neighbor sites is constant $(\Omega)$. The central pair of sites is allowed to have a larger coupling constant, $\Omega^{s p}>\Omega$. This very simple system was considered in the literature (called the "multimer" model [40, 41]) as a prototype model for the PSII RC, and is shown schematically in Fig. 1. Despite its simplicity (currently more complicated models for the ET have been introduced [42, 43]), we believe it contains the essence of the process we are modeling. Later, in Sections III and IV, we will show that the results which follow from our model maintain their validity in a large range of parameters when more realistic models and thermal effects are considered.

The asymmetry in our model arises only from the different coupling strengths with the sinks, which can be understood as representing continuum electron energy spectra. These electron environments are characterized by the transition rates to the left and to the right branches of the system. We also choose symmetric initial conditions for sites 1 and 2 :

$$
\rho(0)=\frac{1}{2}(|1\rangle+|2\rangle)(\langle 1|+\langle 2|) .
$$

(Similar results can be obtained for symmetric mixed state, $\rho(0)=\frac{1}{2}(|1\rangle\langle 1|+| 2\rangle\langle 2|)$.)

An effective non-Hermitian Hamiltonian can be constructed as in [36] by coupling the system to two different continuum electron reservoirs. The probability flow into these continua 


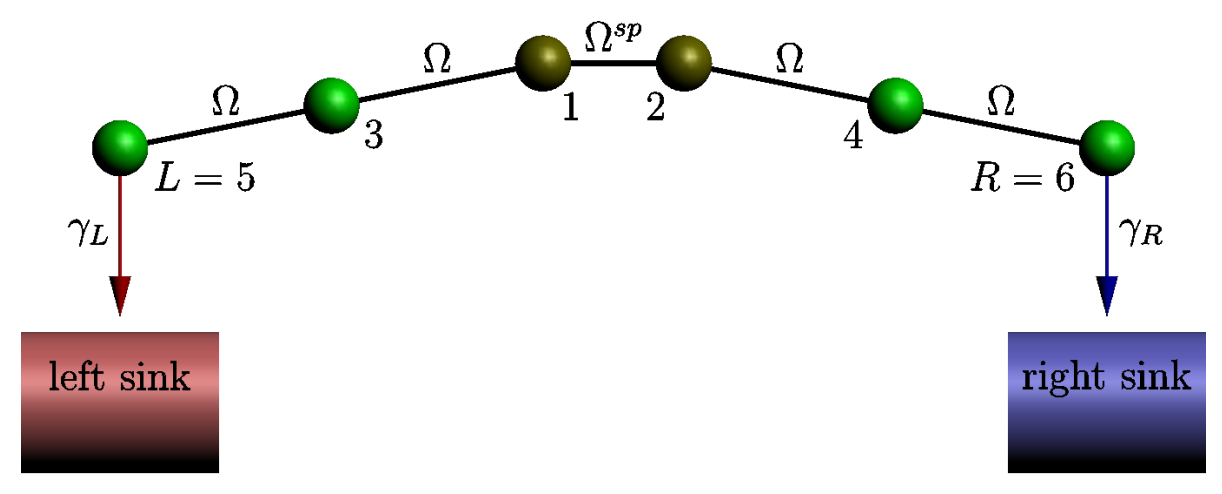

FIG. 1: Multimer model for the PSII RC. The six sites have equal energies, $E_{0}=0$, and equal coupling constants, $\Omega$. The pair $1-2$ is characterized by the coupling constant, $\Omega^{s p}>\Omega$. Left and right ends of the chain are connected to the left and right sinks by the coupling constants, $\gamma_{L}$ and $\gamma_{R}$, that represent couplings to the left and right continuum electron environment.

is analyzed with an effective non-Hermitian Hamiltonian, $\mathcal{H}$,

$$
\mathcal{H} \equiv H_{0}-i \gamma_{L} W_{L}-i \gamma_{R} W_{R}=\left(\begin{array}{cccccc}
0 & \Omega^{s p} & \Omega & 0 & 0 & 0 \\
\Omega^{s p} & 0 & 0 & \Omega & 0 & 0 \\
\Omega & 0 & 0 & 0 & \Omega & 0 \\
0 & \Omega & 0 & 0 & 0 & \Omega \\
0 & 0 & \Omega & 0 & -i \gamma_{L} / 2 & 0 \\
0 & 0 & 0 & \Omega & 0 & -i \gamma_{R} / 2
\end{array}\right)
$$

where $H_{0}$ is the Hamiltonian of the closed system, and $W_{L, R}$ take into account the coherent dissipation. As one can see, in the site basis this corresponds to add imaginary terms to the end sites, $|L\rangle$ and $|R\rangle$, describing the loss of electron probability to the sinks.

The eigenvalues of $\mathcal{H}$ are complex numbers, $E^{(k)}-i \Gamma^{(k)} / 2$, where $\Gamma^{(k)}$ are the decay widths and the evolution is described by the von Neumann equation,

$$
\frac{d \rho}{d t}=-\frac{i}{\hbar}\left(\mathcal{H} \rho-\rho \mathcal{H}^{\dagger}\right) \text {. }
$$

We also introduce the parameters, $\kappa_{L(R)}$ and $q$,

$$
\kappa_{L(R)} \equiv \frac{\gamma_{L(R)}}{2 \Omega}, \quad q \equiv \frac{\kappa_{L}}{\kappa_{R}},
$$

and the efficiencies of the ET to the sinks through the left $(|L\rangle$ site) and the right $(|R\rangle$ site) 
branches during time, $T$ :

$$
\eta_{L(R)}(T)=\frac{\gamma_{L(R)}}{\hbar} \int_{0}^{T} d t\langle L(R)|\rho(t)| L(R)\rangle .
$$

As shown in [35], two superradiant transitions with the corresponding formation of two superradiant (SR) states, are expected to occur, at

$$
\left\{\begin{array}{llll}
\left(S T_{L}\right) & \frac{\gamma_{L}}{2 \Omega} \simeq 1, & \Longrightarrow & \kappa_{L} \simeq 1 \\
\left(S T_{R}\right) & \frac{\gamma_{R}}{2 \Omega} \simeq 1, & \Longrightarrow & \kappa_{L} \simeq q
\end{array}\right.
$$

Strictly speaking, in [35] the ST has been found under the conditions of a very large number of sites $N \gg 1$, and $\Omega^{s p}=\Omega$. We checked that the STs occur even for small $N$ values and in a large range of $\Omega^{s p} \neq \Omega$.

Let us analyze the physical picture in which STs can be seen. A small coupling with the continuum typically produces level broadening, that is all levels equally acquire a width proportional to the strength of the opening. This 'perturbative' argument is valid up to a critical strength: when the widths of neighboring levels overlap a 'segregation' occurs. In other words one energy level continues to have a width proportional to the opening (SR state) while all other levels (subradiant states) are characterized by a decay width proportional to the inverse of the opening strength. This sharp transition has been called superradiant transition, in analogy with the Dicke superradiance, since the SR state owns a width $N$ times larger than the average width (if $N$ is the number of levels) and it decays $N$ time faster than the other states. On the contrary, the subradiants states, in the limit of very large opening strength, loose their widths and they do not decay at all. As shown in Ref. [35], increasing $\kappa_{L}$ at fixed $q$ produces two STs, that can be observed by two peaks in the average width of the $N-2$ subradiant states. (See Fig. 2a.)

We now discuss the efficiency of the ET to the sinks under the condition that the coupling to the left, $\gamma_{L}$, is always larger than the coupling to the right, $\gamma_{R}$. One might expect that the sink with the stronger coupling (stronger probability per unit of time to escape the chain) will be the most efficient. But what happens in this quantum system is more complicated. Indeed, Fig. 2(b) shows that the unbalanced efficiency, $\eta_{L}-\eta_{R}$, as a function of the coupling strength, $\kappa_{L}$, takes almost all values between -1 and 1 , with two maxima close to the STs. In other words, close to the left ST, $\left(S T_{L}, \kappa_{L} \simeq 1\right)$, the ET efficiency has a maximum 

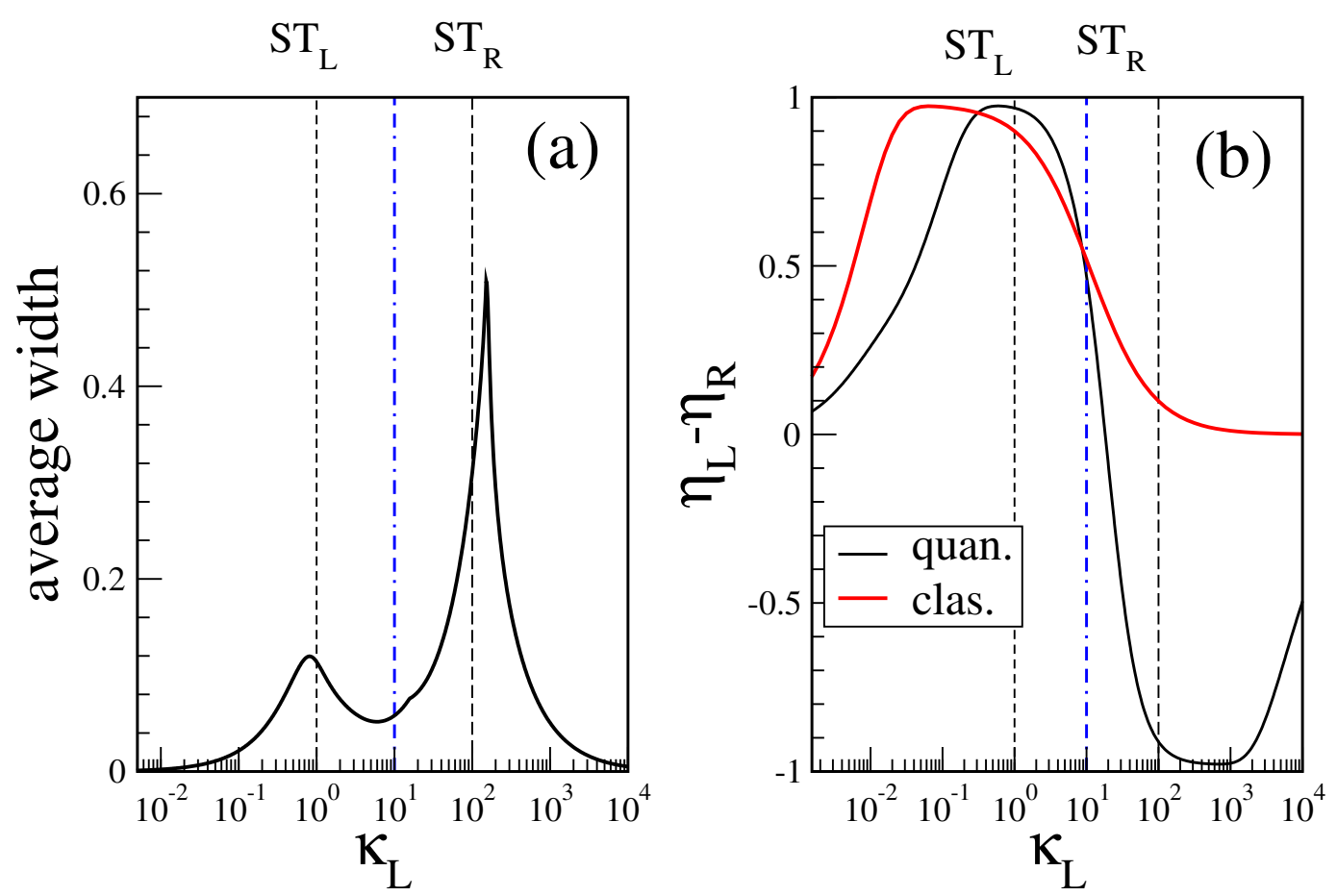

FIG. 2: (Color online) a) Average energy width of the $N-2$ eigenstates which do not become superradiant as a function of the effective coupling strength, $\kappa_{L}$, at fixed $q=\kappa_{L} / \kappa_{R}=100$. Average width has also been renormalized by the average energy distance between levels, $D \simeq \Omega$. b) Unbalanced left-right efficiency, $\eta_{L}-\eta_{R}$, as a function of the effective coupling strength, $\kappa_{L}$, for the quantum case (black lower) and for the classical case (red upper). Here $\Omega=100 \mathrm{~cm}^{-1}$, $\Omega^{s p}=200 \mathrm{~cm}^{-1}$. The efficiencies have been obtained by integrating over $T=20 \mathrm{ps}$. In both panels, the vertical dashed lines represent, respectively, the left and the right STs, while the dashed-dotted central line indicates the switching line, see text.

through the left branch, $\left(\eta_{L} \simeq 1, \eta_{R} \simeq 0\right)$, while close to the right ST, $\left(S T_{R}, \kappa_{L} \simeq q\right)$, the ET efficiency has a maximum at the right branch, $\left(\eta_{L} \simeq 0, \eta_{R} \simeq 1\right)$. This is an unexpected result since the whole picture has been obtained under the condition $\kappa_{L} \gg \kappa_{R}$, namely at the fixed ratio, $q=\kappa_{L} / \kappa_{R}=100 \gg 1$.

Therefore, the whole system can act as a probability switch even if the left coupling is much larger than the right coupling. How is this possible? Before explaining these results and their intrinsic quantum nature, we consider the "classical" behavior of this ET model. The classical dynamics can be modeled considering an incoherent hopping among the sites, thus described by a classical master equation with only diagonal density matrix elements 
(populations):

$$
\frac{d \rho_{i i}}{d t}=\sum_{k}\left(T_{i k} \rho_{k k}-T_{k i} \rho_{i i}\right)-\left(\frac{\gamma_{L}}{\hbar} \delta_{L i}+\frac{\gamma_{R}}{\hbar} \delta_{R i}\right) \rho_{i i},
$$

where $\rho_{i i}$ is the probability at the $i$-th site, $T_{i k}=\left(H_{0}\right)_{i k} / \hbar$ is the transition rate from the $k$-th to the $i$-th sites, and the last two terms represent the flow of probability through the left(L) and right(R) sinks.

The results of the classical dynamics, for the same model and symmetric initial conditions, $\rho_{11}(0)=\rho_{22}(0)=1 / 2$, are shown in Fig. $2 \mathrm{~b}$ (red curve). They demonstrate the absence of a switch of transmission from the left to the right branch. Indeed, one always finds $\eta_{L}>\eta_{R}$, and $\eta_{L} \simeq \eta_{R} \simeq 1 / 2$, for large values of $\kappa_{L}$. Up to some extent, this is in agreement with the intuitive interpretation: if the coupling strengths to both sinks are extremely strong, particles will be absorbed by both sinks with the same efficiency. We can use these results to define "classical transport" as one occurring through the most strongly coupled branch, and "quantum transport" as one occurring through the weaker coupled branch.

These results immediately raise the following two questions: How it can happen that the ET occurs through the weaker coupled branch? Is it possible to estimate analytically the "switching point", $\kappa_{L}^{s w}$, located between two STs at which $\eta_{L} \simeq \eta_{R}$ ?

To answer both questions, we investigated the structure (localization and decay width) of the eigenfunctions of the effective non-Hermitian Hamiltonian. In the region between two STs where the switching occurs, there is only one SR state. Even if its width is very large, it becomes strongly localized around the left sink, leaving other subradiant states approximately extended with no overlap with the left sink (See Appendix A ). This mechanism stops the ET through the left branch and simultaneously induces the ET through the right branch.

Let us analytically estimate the critical value, $\kappa_{L}^{s w}$, at which the switching from left to right occurs. Assuming that the switching occurs when the partial decay width to the left, $\Gamma_{L}$, and to the right, $\Gamma_{R}$, are equal, and since between the two STs, $\Gamma_{L} \propto 1 / \kappa_{L}$ and $\Gamma_{R} \propto \kappa_{L} / q$ (see Appendix B and Ref. [44]) one gets,

$$
\frac{1}{\kappa_{L}} \simeq \frac{\kappa_{L}}{q} \quad \Rightarrow \quad \kappa_{L}^{s w} \simeq \sqrt{q} .
$$

When the condition (8) is satisfied, the unbalanced ET efficiency is approximately zero. This is verified directly in Fig. $2 \mathrm{~b}$, where the theoretical dashed-dotted vertical line, $\kappa_{L}^{s w} \simeq \sqrt{q}$, is in a good agreement with the switching point at which the unbalanced ET efficiency 


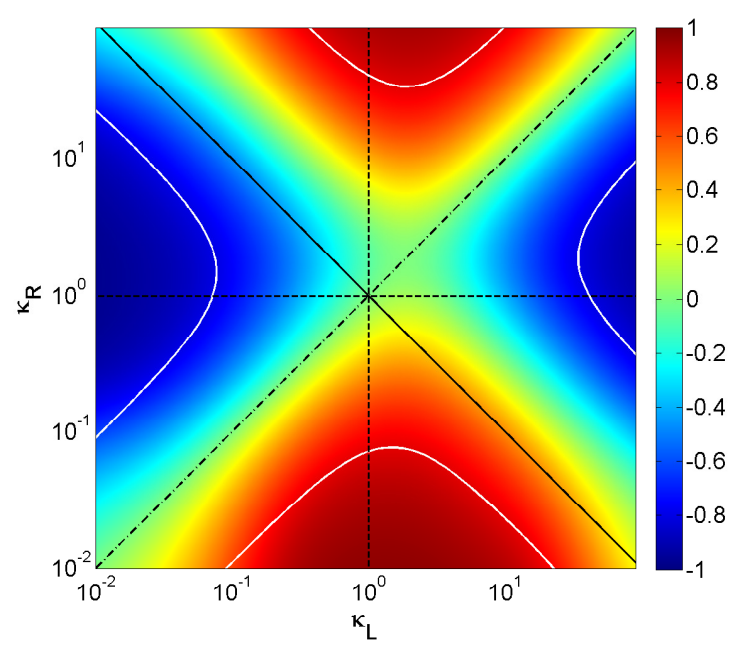

(a)

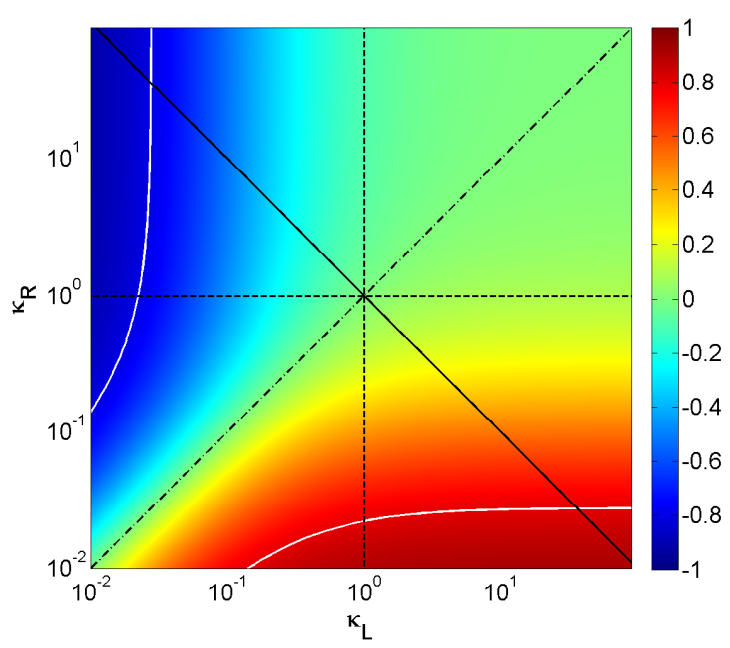

(b)

FIG. 3: The $2 \mathrm{~d}$ contour plot, $\eta_{L}-\eta_{R}$, as a function of $\kappa_{L}=\gamma_{L} / 2 \Omega$ and $\kappa_{R}=\gamma_{R} / 2 \Omega$ in log-log scale for the quantum (a) and the classical case (b). The vertical and horizontal lines are, respectively, the $S T_{L}$ and $S T_{R}$. The two diagonals are the symmetry line $\kappa_{L}=\kappa_{R}$ (dashed) and the switching line $\kappa_{L}=1 / \kappa_{R}$ (full). White curves represent the $90 \%$ of the ratio between the two efficiencies $\left(\eta_{L} / \eta_{R}=9\right.$ or $\left.\eta_{R} / \eta_{L}=9\right)$.

becomes zero. Note that in our case the same condition in Eq. (8) also defines the minimal decay width between the two STs, see Fig. 2 a.

One could ask whether these results are due to the strong asymmetry $(q=100)$ used above. The answer can be extracted from the "phase diagram" of Fig. 3, in which the unbalanced efficiency is shown for all values of $\kappa_{L}$ and $\kappa_{R}$, and for both the quantum case (left panel) and the classical case (right panel), where the regions of transport to the left have been indicated by red color and the transport to the right branch by the blue color. While the classical picture shows that to switch between the red and the blue regions it is necessary to cross the symmetry line, $\kappa_{L}=\kappa_{R}$, in the quantum world a further possibility is given by crossing the curve, $\kappa_{L}=1 / \kappa_{R}$. 


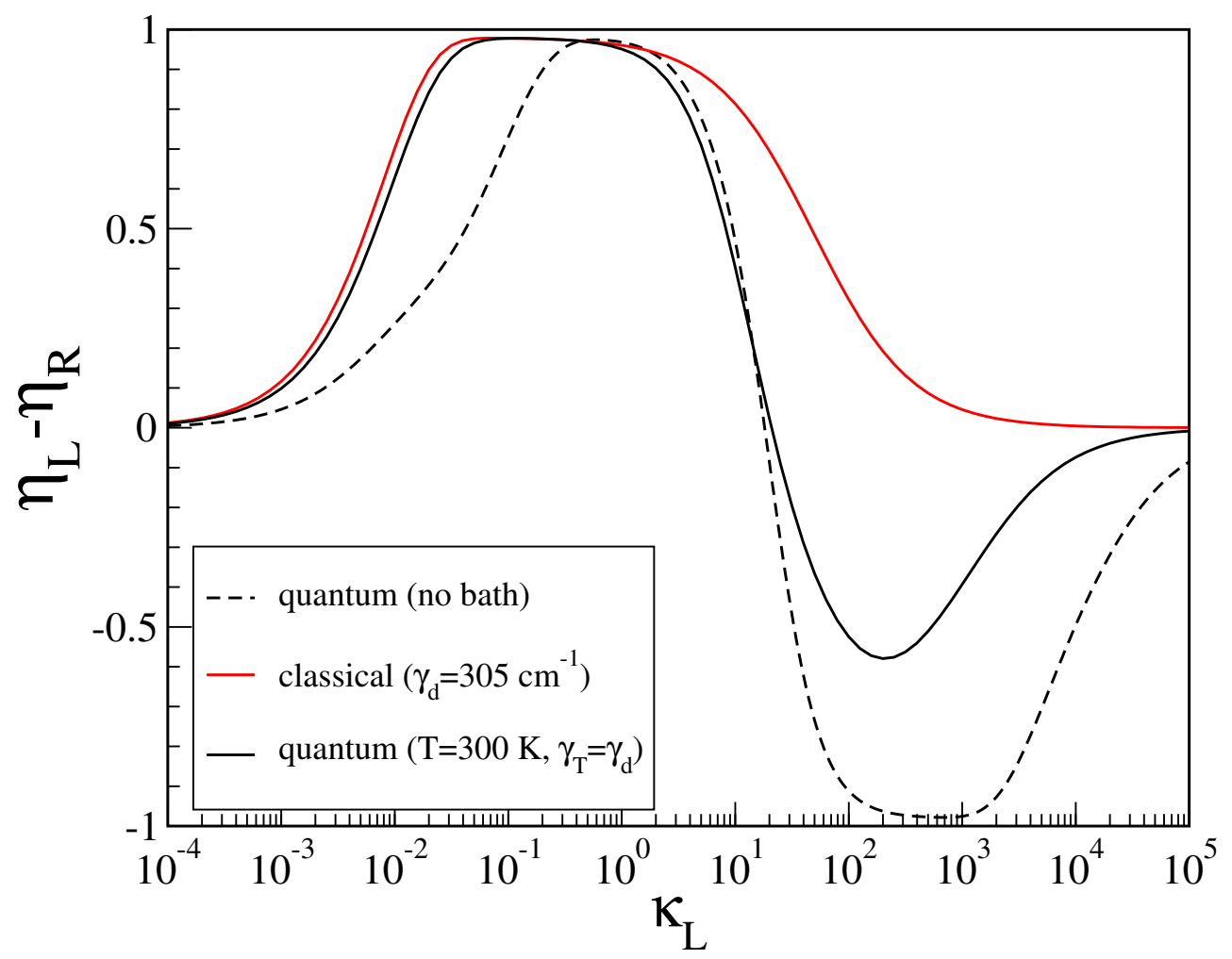

FIG. 4: (Color online) Effects of a thermal bath. Unbalanced left-right ET efficiency, $\eta_{L}-\eta_{R}$, as a function of the effective coupling strength, $\kappa_{L}$. Black full line stands for quantum transport in presence of a thermal bath at $T=300 \mathrm{~K}, \omega_{c}=150 \mathrm{~cm}^{-1}, E_{R}=35 \mathrm{~cm}^{-1}$, so to have a homogeneous line broadening $\gamma_{T}=305.7 \mathrm{~cm}^{-1}$. Dashed line is the quantum calculation did before (microcanonical). Red line stands for the classical master equation Eq. 7, with the semiclassical rates, Eq. 10, and $\gamma_{d}=\gamma_{T}=305.7 \mathrm{~cm}^{-1}$. Here $\Omega=100 \mathrm{~cm}^{-1}, \Omega^{s p}=200 \mathrm{~cm}^{-1}$, and $q=$ $\kappa_{L} / \kappa_{R}=100$ fixed. Left and right ET efficiencies have been obtained by integrating over 20 ps.

\section{EFFICIENCY OF THE ET IN THE PRESENCE OF A THERMAL BATH.}

To demonstrate the robustness of the approach described above, consider the interaction of our system with a phonon bath at finite temperature. We use the thermal bath as in [45] whose dynamics is described by the Lindblad master equation in the Born-Markov and secular approximations. More realistic models for the thermal bath can be found in the literature [42, 43]. This simpler approach has already been used to describe the FMO complex in a very similar framework [36, 45]. Note that this approach leads to the relaxation of the populations, $\rho_{k k}$, to the Gibbs distribution. 
We use the Lindblad-type master equation in the form,

$$
\frac{d \rho}{d t}=-\frac{i}{\hbar}\left(\mathcal{H} \rho-\rho \mathcal{H}^{\dagger}\right)+L_{p}(\rho)
$$

where the action of the Lindblad operator, $L_{p}(\rho)$, on $\rho$ is described by Eq. (5) of Ref. [45]. (See Appendix C .) In particular, we choose an exponential spectral density dependent on two parameters, the reorganization energy, $E_{R}$, and the cutoff frequency, $\omega_{c}$, to be considered together with the temperature, $T$, of the bath.

The thermal bath (interaction with phonons) produces an homogeneous line broadening [46], proportional to both temperature and reorganization energy, and inversely proportional to the cut-off frequency 47 ]

$$
\gamma_{T}=2 \pi\left(\frac{k T}{\hbar}\right)\left(\frac{E_{R}}{\hbar \omega_{c}}\right)
$$

In Fig. 4, we plot the unbalanced left-right ET efficiency as a function of the coupling, $\kappa_{L}$, at $T=300 \mathrm{~K}$, and reorganization energy and cut-off frequency chosen in order to have an homogeneous line broadening $\gamma_{T}=305.7 \mathrm{~cm}^{-1}$ (full black curve).

As one can see, the first effect is that the quantum switching due to the superradiance is weakened but not suppressed by the thermal bath (compare with the dashed curve which represents the same quantity in absence of the thermal bath for the same value of $q=$ $\left.\kappa_{L} / \kappa_{R}=100\right)$.

To have a close comparison with the classical model we consider the same classical master equation as before, Eq. (7), but with the transition rates, computed semiclassically as in [36, 46],

$$
T_{i k}=\frac{2 \Omega_{i k}^{2}}{\hbar \gamma_{d}}\left(1+\frac{\Delta E_{i k}^{2}}{\gamma_{d}^{2}}\right)^{-1},
$$

where $\Omega_{i k}$ represents the energy coupling between the $i$-th and the $k$-th sites, the $\Delta E_{i k}$ are the energy differences between the two sites, and $\gamma_{d}$ is the dephasing energy [46].

To have a close comparison we put the dephasing energy $\gamma_{d}=\gamma_{T}$. Results obtained from the classical master equation with the semiclassical rates Eq. (11) are shown in Fig. 4 as a red curve.

As one can see, the switching is absent in the classical model since the incoherent hopping transport gives at most $\eta_{R} \approx \eta_{L}=1 / 2$. The most interesting result is that the presence of thermal bath has the opposite effects on classical and quantum transport: the thermal bath weakens only the quantum transport (transport to the right weakest coupled branch) while it leaves mainly unaffected the classical transport (left strongest coupled branch). The 

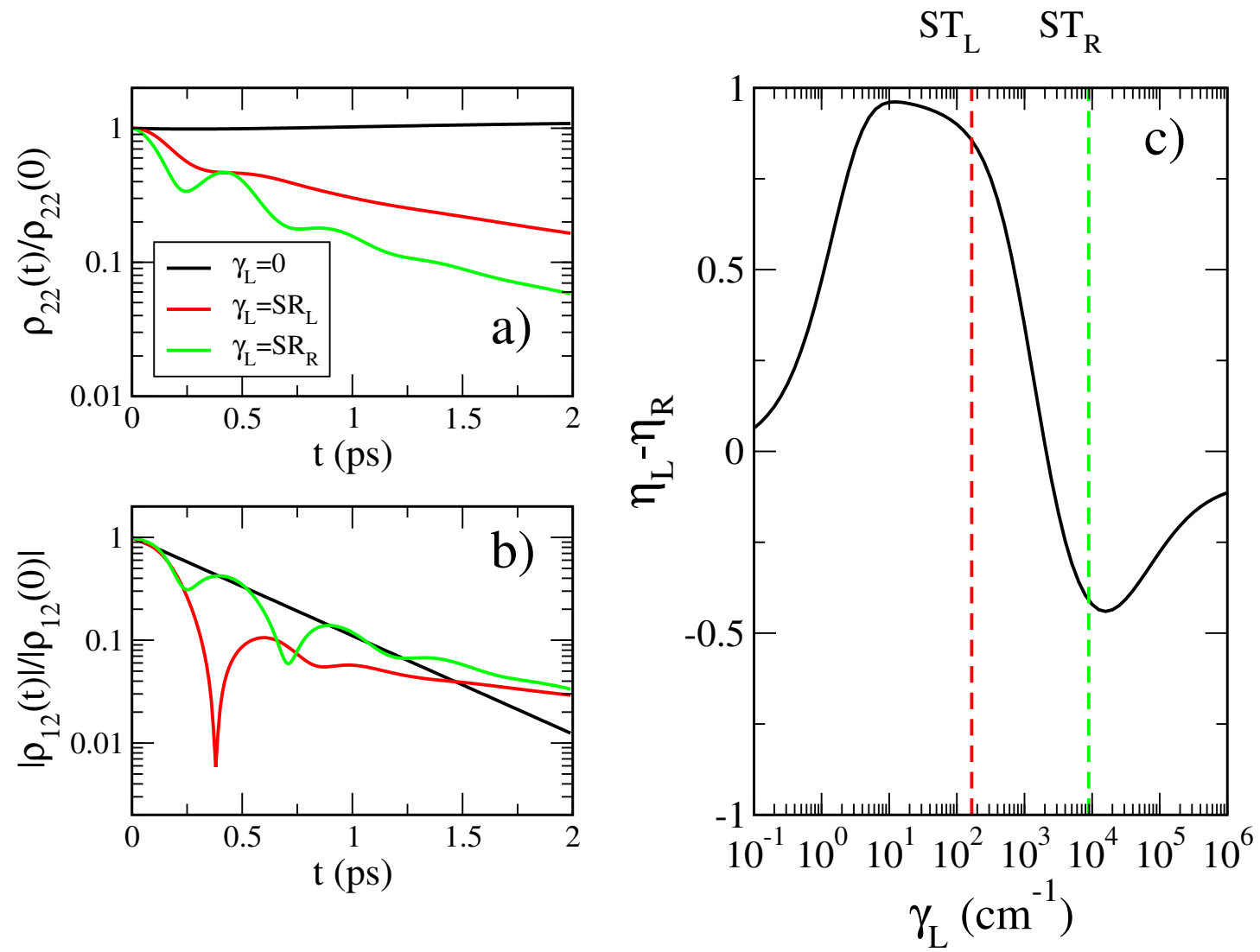

FIG. 5: (Color online) Realistic model of a RC. Left panels: a) Decay of populations in time, b) Decay of coherences in time for fixed ratio, $q=\gamma_{L} / \gamma_{R}=100$, and different values of $\gamma_{L}$, as indicated in the legend. Right panel c): Unbalanced average left-right ET efficiency, $\eta_{L}-\eta_{R}$, as a function of the effective coupling strength, $\gamma_{L}$, for $300 \mathrm{~K}$. A reorganization energy, $E_{R}=20 \mathrm{~cm}^{-1}$, and cut-off frequency, $\omega_{c}=150 \mathrm{~cm}^{-1}$, have been chosen in order to have an approximate decay of populations and coherences on the time-scale of 1 ps. (See left panels a) and b.)

different sensibility of ET to the dephasing induced by the thermal bath is consistent with the quantum coherent nature of the switch. This opens the interesting possibility to use the switch effect as a witness of quantum coherence in molecular chains.

\section{EFFICIENCY FOR A REALISTIC MODEL.}

Here we apply our approach to a realistic model [48] of the photosystem II reaction center (PSII RC). This system has eight chromophores in the Left-Right subunits: two chlorophylls belonging to the central special pair, two accessory chlorophylls, two pheophytins and two 
peripheral chlorophylls, not relevant since weakly coupled. To match $2 \mathrm{D}$ spectroscopy data, the energy levels in the active branch (left) are not the same as for the inactive branch (right), and range between 15000 and $15555 \mathrm{~cm}^{-1}$. Moreover, the coupling constants among chromophores are not equal for nearest neighbors, and all chromophores couple to each other with strengths varying from 0.12 to $162.2 \mathrm{~cm}^{-1}$. The exact Hamiltonian matrix is not presented here. It appears in Ref. [48]. Due to many differences from the simple model discussed above, one can wonder whether the previously discussed switching effect persists in this realistic model characterized by non-degenerate energy levels, long-range interactions, and absence of exact left-right symmetry [48].

We proceed as before, by attaching the sinks to the pheophytins, designing the effective non-Hermitian Hamiltonian, and calculating the conditions for the left and the right STs (computed from the complex eigenvalues of the non-Hermitian Hamiltonian of this model, indicated as dashed vertical lines in Fig. 5k). As one can see, the maximal left/right ET efficiency roughly peaks near the $S T_{L, R}$.

In order to show that the switch can work at room temperature, we embedded the system in a thermal bath as described above. We note that the bath considered in Eq. (9), without sinks, produces typically uncoupled equations for populations (diagonal matrix elements) and coherences (off-diagonal ones) in the energy basis. In particular, populations relax in time to Gibbs distribution without oscillations, at variance with the experiment [6-14]. Interestingly, the coupling with the sinks produces an effective coupling between populations and coherences. The effect of the dynamics generated by the sinks is shown in Figs. 5a) and b), in which oscillations are clearly observable for $\gamma_{L} \neq 0$, showing that coherences and populations are now coupled.

We therefore use the same thermal bath as in Eq. (9), setting the parameters for reorganization energy, $E_{R}$, and cut-off frequency, $\omega_{c}$, in order to have a decay of both populations and coherences of the order of 1 ps, in agreement with experiments [13]. Our results (shown in Fig. 5 c), demonstrate that the previously observed switching survives in the presence of a thermal bath at room temperature (even though it reduces the unbalanced ET efficiency to the right branch $(\approx 0.5$ compared with the left $\approx 1$ ). 


\section{CONCLUSIONS.}

The analysis of our model for electron transfer, consisting of two branches attached to two asymmetric sinks, revealed two different transport regimes: a classical one, in which the electron transport occurs through the strongest coupled sink, and a quantum one, in which the electron transport occurs through the weakest coupled sink. Varying the coupling strengths in an appropriate way, see Fig. 3a, one can switch from one regime to the other, thus inducing a switching of electron transfer from one branch to the other. This switching is a pure quantum effect, based on the existence of two consecutive superradiance transitions as the couplings vary. The quantum nature of the switching is confirmed by the analysis of the coupling to a thermal bath: only quantum transport is weakened by the thermal bath,

not the classical one. This opens the possibility of using this switching effect to measure the amount of quantum coherence in molecular networks. Analyzing a realistic model of the photosystem II reaction center at room temperature, we have shown that this switching mechanism, being robust to disorder and dephasing, could be observed in natural biological complexes.

\section{ACKNOWLEDGMENTS.}

This work has been supported by Regione Lombardia and CILEA Consortium through a LISA Initiative (Laboratory for Interdisciplinary Advanced Simulation) 2011 grant [link:http://lisa.cilea.it]. Support by the grant D.2.2 2011 (Calcolo ad alte prestazioni) from Universitá Cattolica is also acknowledged. The work by GPB and RTS was carried out under the auspices of the National Nuclear Security Administration of the U.S. Department of Energy at Los Alamos National Laboratory under Contract No. DE-AC52-06NA25396. We also thank Gary Doolen for useful comments.

[1] Barber, J. Photosynthetic energy conversion: natural and artificial. Chem. Soc. Rev. 38, 185196 (2009).

[2] Tachibana, Y., Vayssieres, L. \& Durrant J.R. Artificial photosynthesis for solar water-splitting. Nature Photonics, 6, 511-518 (2012). 
[3] Valov, I., Linn, E., Tappertzhofen, S., Schmelzer, S, van den Hurk, J., Lentz, F. \& Waser R. Nanobatteries in redox-based resistive switches require extension of memristor theory. Nature Communications 4, 1771, (2013).

[4] Smirnov, A., Mourokh, L.G., Ghosh, P.K. \& Nori F. High-efficiency energy conversion in a molecular triad connected to conducting leads. J. Phys. Chem. C 113, 21218-21224 (2009).

[5] Carrara, S. Nano-Bio-Technology and Sensing Chips: New Systems for Detection in Personalized Therapies and Cell Biology. Sensors 10, 526-543 (2010).

[6] Hu X., Ritz T., Damjanovich A. \& Schulten, K. Pigment Organization and Transfer of Electronic Excitation in the Photosynthetic Unit of Purple Bacteria. J. Phys. Chem. B 101, 3854-3871, (1997).

[7] Brixner, T., Stenger, J., Vaswani, H.M., Cho, M., Robert E. Blankenship, R.E. \& Fleming, G.R. Two-dimensional spectroscopy of electronic couplings in photosynthesis. Nature 434, 625-628 (2005).

[8] Lee, H., Cheng, Y-C. \& Fleming, G.R. Coherence Dynamics in Photosynthesis: Protein Protection of Excitonic Coherence. Science 316, 1462-1465 (2007).

[9] Engel, G.S., Calhoun, T.R., Read, E.L., Ahn, T.K., Man., T., Cheng, Y.C., Robert E. Blankenship, R.E. \& Fleming, G.R. Evidence for wavelike energy transfer through quantum coherence in photosynthetic systems. Nature 446, 782-786 (2007).

[10] Raszewski, G., Diner, B.A., Schlodder, E. \& Renger, T. Spectroscopic Properties of Reaction Center Pigments in Photosystem II. Core Complexes: Revision of the Multimer Model. Biophys. J. 95, 105-119 (2008).

[11] Panitchayangkoon, G., Hayes, D., Fransted, K.F., Caram, J.R., Harel, E., Wen, J., Blankenshipb, R.E. \& Engel, G.S. Long-lived quantum coherence in photosynthetic complexes at physiological temperature. Proc. Natl. Acad. Sci. U.S.A. 107, 12766-12770, (2010).

[12] Collini,E., Wong,C.Y., Wilk, K.E., Curmi, P.M.G., Brumer, P. \& Scholes, G.D. Coherently wired light-harvesting in photosynthetic marine algae at ambient temperature. Nature 463, 644-647, (2010).

[13] Westenhoff, S., Palecek, D., Edlund, P., Smith, P. \& D. Zigmantas, D. Coherent Picosecond Exciton Dynamics in a Photosynthetic Reaction Center. J. Am. Chem. Soc. 134, 16484-16487, (2012).

[14] Wong, C.Y., Alvey, R.M., Turner, D.B., Wilk, K.E., Bryant, D.A., Curmi, P.M., Silbey, 
R.J. \& Scholes, G.D. Electronic coherence lineshapes reveal hidden excitonic correlations in photosynthetic light harvesting. Nature Chemistry 4, 396-404 (2012).

[15] Ishizaki, A. \& Fleming, G.R. Theoretical examination of quantum coherence in a photosynthetic system at physiological temperature. Proc. Natl. Acad. Sci. U.S.A. 106, 17255-17260 (2009).

[16] Cheng, Y.-C. \& Fleming, G.R. Dynamics of Light Harvesting in Photosynthesis. Annu. Rev. Phys. Chem. 60, 241-262 (2009).

[17] Scholes, G.D., Fleming, G.R., Olaya-Castro, A. \& van Grondelle, R. Lessons from nature about solar light harvesting. Nature Chemistry 3 , 763-774 (2011).

[18] Strumpfer, J., Sener, M. \& Schulten, K. How Quantum Coherence Assists Photosynthetic Light-Harvesting. J. Phys. Chem. Lett. 3, 536-542 (2012).

[19] Dawlaty, J.M., Ishizaki, A., De, A.K. \& Fleming, G.R. Microscopic quantum coherence in a photosynthetic-light-harvesting antenna. Philos. Trans. Roy. Soc. A 370, 3672-3691 (2012).

[20] Kassal, I. Yuen-Zhou, J. \& Rahimi-Keshari, S. Does Coherence Enhance Transport in Photosynthesis? J. Phys. Chem. Lett. 4, 362-367 (2013).

[21] Scherer, P.O.J. \& Fischer, S.F. Long-Range Electron Transfer within the Hexamer of the Photosynthetic Reaction Center Rhodopseudomonas viridis, J. Phys. Chem., 93, 1633-1637 (1989).

[22] Giorgi, L.B., Nixon, P.J., Merry, S A.P., Joseph, D.M., Durrant, J.R., De Las Rivas, J., Barber, J., Porter, G. \& Klug, D.R. Comparison of Primary Charge Separation in the Photosystem II Reaction Center Complex Isolated from Wild-type and D1-130 Mutants of the Cyanobacterium Synechocystis PCC 6803, J. Biol. Chem., 271, 2093-2101 (1996).

[23] Dorlet, P., Xiong, L., R.T. Sayre, R.T. \& Un, S. High Field EPR Study of the Pheophytin Anion Radical in Wild Type and D1-E130 Mutants of Photosystem II in Chlamydomonas reinhardtii, J. Biol. Chem. 276, 22313-22316 (2001).

[24] Xiong, L., Seibert, M., Gusev, A.V., Wasielewski, M.R., C. Hemann, C., Hille \& Sayre, R.T. Substitution of a Chlorophyll into the Inactive Branch Pheophytin-Binding Site Impairs Charge Separation in Photosystem II. J. Phys. Chem. B 108, 16904-16911 (2004).

[25] Rappaport, F., Cuni, A., Xiong, L., R. Sayre, R. \& Lavergne, J. Charge Recombination and Thermoluminescence in Photosystem II, Biophysical Journal 88, 1948-1958 (2005).

[26] Pincak, R. \& Pudlak, M. Noise breaking the twofold symmetry of photosynthetic reaction 
centers:Electron transfer. Phys. Rev. E 64, 031906 (2001).

[27] Pudlak, M. Primary charge separation in the bacterial reaction center: Validity of incoherent sequential model. J. Chem. Phys. 118, 1876 (2003).

[28] Lakhno, V.D. Oscillations in the primary charge separation in bacterial photosynthesis. Phys. Chem. Chem. Phys. 4 , 2246-2250 (2002).

[29] Lakhno, V.D. Dynamical Theory of Primary Processes of Charge Separation in the Photosynthetic Reaction Center. J. Biol. Phys. 31, 145-159 (2005).

[30] Pudlak, M. \& Pincak, R. Electronic pathway in reaction centers from Rhodobacter sphaeroides and Chloroflexus aurantiacus. J. Biol. Phys. 36, 273-289 (2010).

[31] Novoderezhkin, V.I., Dekker, J.P. \& van Grondelle, R. Mixing of Exciton and Charge-Transfer States in Photosystem II Reaction Centers: Modeling of Stark Spectra with Modified Redfield Theory. Biophys. J. 93, 1293-1311 (2007).

[32] Abramavicius, D. \& Mukamel, S. Energy-transfer and charge-separation pathways in the reaction center of photosystem II revealed by coherent two-dimensional optical spectroscopy. $J$. Chem. Phys. 133, 184501 (2010).

[33] Abramavicius, D. \& Mukamel, S. Quantum oscillatory exciton migration in photosynthetic reaction centers. J. Chem. Phys. 133, 064510 (2010).

[34] Superradiance transition in one-dimensional nanostructures: An effective non-Hermitian Hamiltonian formalism. Celardo, G. L., Kaplan, L., Phys. Rev. B, 79, 155108 (2009).

[35] Celardo, G.L., Smith, A.M., Sorathia, S., Zelevinsky, V.G., Sen'kov, R.A. \& Kaplan, L. Transport through nanostructures with asymmetric coupling to the leads. Phys. Rev. B 82, $165437(2010)$

[36] Celardo, G.L., Borgonovi, F., Merkli, M., Tsifrinovich, V.I. \& Berman, G.P. Superradiance Transition in Photosynthetic Light-Harvesting Complexes. J. Phys. Chem. C 116 , 22105$22111(2012)$.

[37] Spano, F.C \& Mukamel, S. Superradiance in molecular aggregates, J. Chem. Phys. 91 (1989). Monshouwer, R. Abrahamsson, M. Van Mourik, F. \& and van Grondelle, R. Superradiance and Exciton Delocalization in Bacterial Photosynthetic Light-Harvesting Systems. J. Phys. Chem. B 101, 7241-7248 (1997)

[38] Auerbach, N. \& Zelevinsky, V. Super-radiant dynamics, doorways and resonances in nuclei and other open mesoscopic systems. Rep. Prog. Phys. 74 106301-106335 (2011). 
[39] Lloyd, S., Mohseni, M. Symmetry-enhanced supertransfer of delocalized quantum states. New J. Phys. 12, 075020 (2010).

[40] Durrant, J.R. et al., A multimer model for P680, the primary electron donor of photosystem II. Proc. Natl. Acad. Sci. U.S.A. 92, 4798-4802 (1995).

[41] Leegwater, J.A., Durrant, J.R. \& Klug, D.R. Exciton Equilibration Induced by Phonons: Theory and Application to PS II Reaction Centers. J. Phys. Chem. B 101, 7205-7210 (1997).

[42] Palmieri, B., Abramavicius. D. \& Mukamel, S. Lindblad equations for strongly coupled populations and coherences in photosynthetic complexes. J. Chem. Phys. 130, 240512 (2009).

[43] Ishizaki, A. \& Fleming, G.R. On the Interpretation of Quantum Coherent Beats Observed in Two-Dimensional Electronic Spectra of Photosynthetic Light Harvesting Complexes. J. Phys. Chem. B 115, 6227-6233 (2011).

[44] Celardo, G.L., Biella, A., Kaplan, L. \& Borgonovi, F. Interplay of superradiance and disorder in the Anderson model. Fortschr. Phys. 61, 250-260 (2013).

[45] Mohseni, M., Rebentrost, P., Lloyd, S. \& Aspuru-Guzik, A. Environment-assisted quantum walks in photosynthetic energy transfer. J. Chem. Phys. 129, 174106 (2008).

[46] Leegwater, J.A. Coherent versus Incoherent Energy Transfer and Trapping in Photosynthetic Antenna Complexes. J. Phys. Chem. 100, 14403-14409 (1996).

[47] Rebentrost, P., Mohseni, M., Kassal, I., Lloyd, S. \& Aspuru-Guzik, Environment-assisted quantum transport. New J. Phys. 11, 033003 (2009).

[48] Lewis, K.L.M., Fuller, F.D., Myers, J.A., Yocum, C.F., S. Mukamel, S., Abramavicius, D. \& Ogilvie, J.P. Simulations of the Two-Dimensional Electronic Spectroscopy of the Photosystem II Reaction Center. J. Phys. Chem. A 117, 34-41 (2013). 

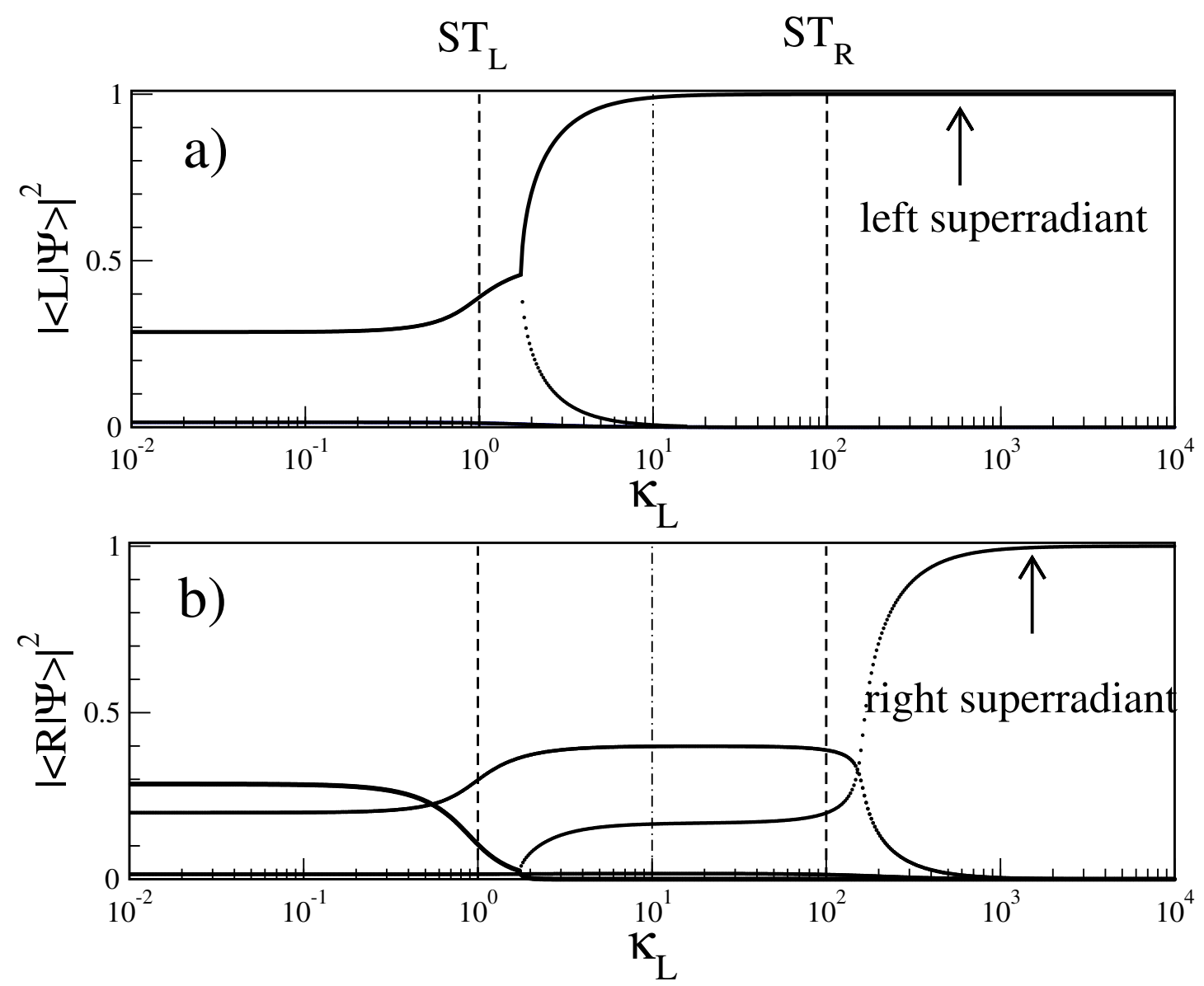

FIG. 6: a) Probability to be at the left end $|\langle L \mid \Psi\rangle|^{2}$, for all energy eigenstates $|\Psi\rangle$. b) Probability to be at the right end $|\langle R \mid \Psi\rangle|^{2}$, for all energy eigenstates. Here $q=100$.

\section{APPENDIX A: PARTICIPATION RATIO}

In Fig. 6a, we show the probabilities to occupy the end sites $(|L\rangle,|R\rangle)$ for all energy eigenstates. As one can see, on approaching the left transition, $S T_{L}$, the superradiant state, $|S R\rangle$, increases its probability to occupy the left end, and this probability becomes one at the switching point. Of course, at the same point the probability to be at the left end of the other states becomes zero, so that ET to the left sink is completely inhibited, and ET switches to the right sink.

One can also observe that after the $S T_{R}$, the same effect occurs at very large coupling thus inhibiting also the ET to the right end. This is at variance with the classical behavior, where for very large coupling one gets, $\eta_{L}=\eta_{R}=1 / 2$.

In order to study the localization of the eigenstates, $|\Psi\rangle$, we consider their participation 

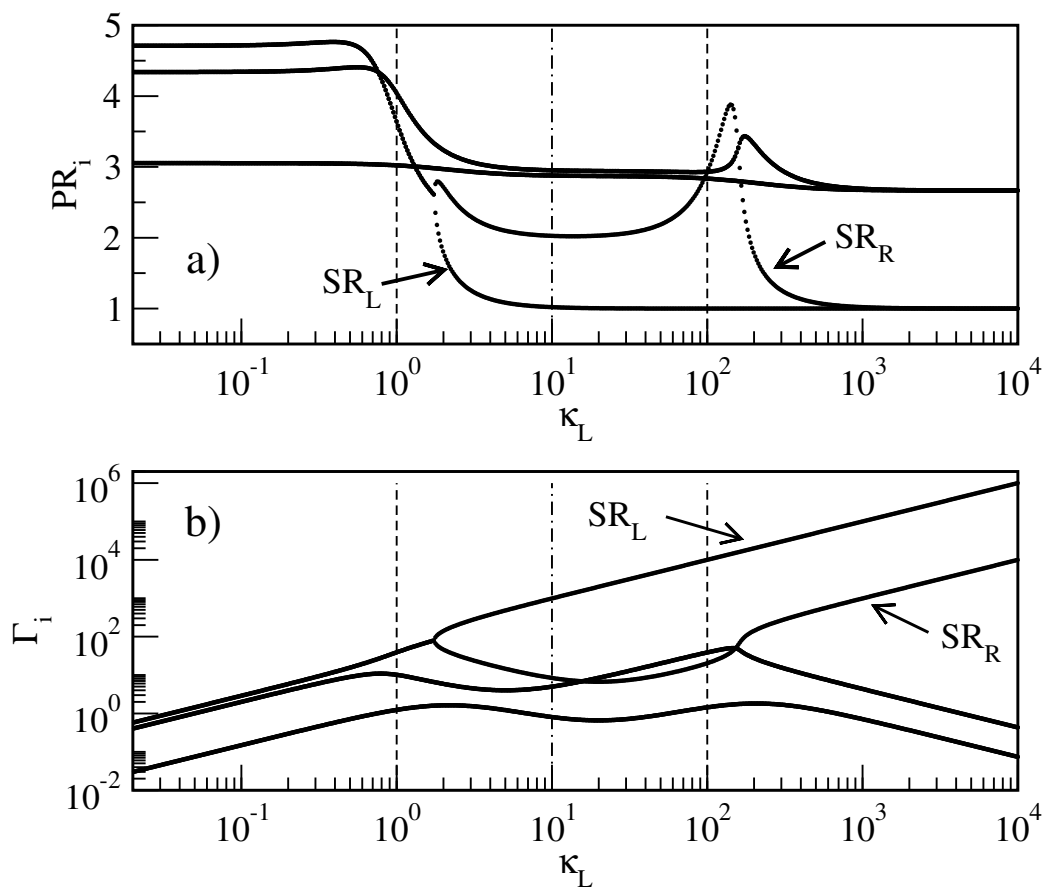

FIG. 7: a) Participation ratio versus $\kappa_{L}$ for all states. b): Decay width versus $\kappa_{L}$ for all states. Here $q=100$.

ratio in the site basis $|n\rangle$,

$$
P R=\frac{1}{\sum_{n}|\langle n \mid \Psi\rangle|^{4}}
$$

In Fig. $7 \mathrm{a}$ we show the PR of all energy eigenstates. As one can see, for $\kappa_{L}<1$ most of the states are approximately delocalized over the whole system (the maximal $P R$ corresponds to the total number of sites, 6). For $1<\kappa_{L}<q$ both superradiant and subradiant states tend to localize. However, while the $P R$ of the left superradiant state $S R_{L}$ becomes $\approx 1$ at the switching point $\kappa_{L} \sim \sqrt{q}$ (complete localization), the $P R$ of the other states does not decrease below $\approx 3$, which means that they are approximately extended over the system, thus allowing the ET.

Even if the left superradiant state becomes extremely localized immediately after the $S T_{L}$, its width is very large. (See Fig. 7b.) This competition between localization and decay width determines the switching point of ET. 


\section{APPENDIX B: THE CRITICAL SWITCHING POINT}

Let us first define the left/right partial width as follow,

$$
\Gamma_{L, R}=\gamma_{L, R} \sum_{k}\left\langle k\left|W_{L, R}\right| k\right\rangle,
$$

where the sum is taken over the subradiant states, $|k\rangle$. Let us also consider the range of parameters between the two STs, namely $1<\kappa_{L}<q$, and the effective Hamiltonian,

$$
\mathcal{H}=-i \gamma_{L} W_{L}+\left(H_{0}-i \gamma_{R} W_{R}\right)
$$

where we consider $-i \gamma_{L} W_{L}$ as the unperturbed Hamiltonian, and $H_{0}-i \gamma_{R} W_{R}$, as the perturbation.

Let us consider, for definiteness, the site $R$ as the first site $j=1$ and the left $L$ as the last $j=N$. Eigenvalues for $W_{L}$ are 1 and 0 , the latter $N-1$ times degenerate; the eigenvector correspondent to 1 is $|L\rangle$, since,

$$
W_{L}|L\rangle=|L\rangle
$$

while, as a degenerate basis we can choose the site basis $|j\rangle$, for $j=1, \ldots, N-1$, since $W_{L}|j\rangle=0$.

Eigenvalues and eigenvectors of the $N-1$ degenerate system can be obtained at zero order by solving the eigenvalues problem,

$$
\left\langle k\left|H_{0}-i \gamma_{R} W_{R}\right| j\right\rangle=\epsilon_{k} \delta_{j k} .
$$

Since $H_{0}$ is of the order of $\Omega$, and $\gamma_{R} / \Omega \ll 1$, we can use first order perturbation theory in $\gamma_{R} / \Omega$ to get the eigenvalues,

$$
\epsilon_{q}^{I}=\epsilon_{q}^{0}-i \gamma_{R}\left\langle q\left|W_{R}\right| q\right\rangle
$$

and the eigenvectors,

$$
|q\rangle^{I}=|q\rangle-\frac{i \gamma_{R}}{2 \Omega N} \sum_{q^{\prime} \neq q} \frac{\sin (q \pi / N) \sin \left(q^{\prime} \pi / N\right)}{\sin \left[\left(q+q^{\prime}\right) \pi / N\right] \sin \left[\left(q-q^{\prime}\right) \pi / N\right]}\left|q^{\prime}\right\rangle \equiv|q\rangle-\frac{i \gamma_{R}}{2 \Omega N} \sum_{q^{\prime} \neq q} c_{q, q^{\prime}}\left|q^{\prime}\right\rangle,
$$

where we have chosen the eigenbasis by the restriction of $H_{0}$ to the $N-1$ dimensional space,

$$
\langle k \mid q\rangle=\sqrt{\frac{2}{N}} \sin \left(\frac{\pi k q}{N}\right),
$$

with eigenvalues $\epsilon_{q}^{0}=-2 \Omega \cos (\pi q / N)$. The same eigenvectors can be considered (with the same name) in the $N$-th dimensional space, by simply adding a 0 in the $N$-th component, so to be orthogonal to $|L\rangle$.

To have the eigenstates at first order of the perturbation theory in $\Omega / \gamma_{L}$ and $\gamma_{R} / \gamma_{L}$, one 
should take into account the interaction between $|q\rangle$ and $|L\rangle$, mediated by the perturbation $H_{0}-i \gamma_{R} W_{R}$, so that,

$$
|q\rangle^{I}=|q\rangle-\frac{i \gamma_{R}}{2 \Omega N} \sum_{q^{\prime} \neq q} c_{q, q^{\prime}}\left|q^{\prime}\right\rangle+\frac{\left\langle q\left|H_{0}-i \gamma_{R} W_{R}\right| L\right\rangle}{-i \gamma_{L}-\epsilon_{q}^{0}}
$$

From this one gets,

$$
\begin{aligned}
& \langle R \mid q\rangle^{I}=\langle R \mid q\rangle+O\left(\gamma_{R} / \Omega\right), \\
& \langle L \mid q\rangle^{I}=i \sqrt{\frac{2}{N}} \frac{\Omega}{\gamma_{L}} \sin \left(\frac{\pi q}{N}\right)+O\left(\Omega / \gamma_{L}\right) .
\end{aligned}
$$

From Eq. (18) the partial widths easily follows,

$$
\begin{aligned}
& \Gamma_{R}=\frac{2 \gamma_{R}}{N} \sum_{q} \sin ^{2}(\pi q / N), \\
& \Gamma_{L}=\frac{2 \Omega^{2}}{N \gamma_{L}} \sum_{q} \sin ^{2}(\pi q / N) .
\end{aligned}
$$

Equating Eq. (18) and Eq. (19), one gets,

$$
\gamma_{L} \gamma_{R} \simeq \Omega^{2}
$$

\section{APPENDIX C: LINDBLAD MASTER EQUATION}

The dynamics of the system to second order in the system-bath coupling can be described by the Lindblad master equation in the Born-Markov and secular approximations as

$$
\frac{d \rho}{d t}=-\frac{i}{\hbar}\left(\mathcal{H} \rho-\rho \mathcal{H}^{\dagger}\right)+L_{p}(\rho)
$$

where the superoperator, $L_{p}$, acts on $\rho$ as follow,

$$
\begin{aligned}
L_{p}(\rho) & =\sum_{\omega, m} \gamma(\omega)\left[A_{m}(\omega) \rho A_{m}^{\dagger}(\omega)-\frac{1}{2} A_{m}^{\dagger}(\omega) A_{m}(\omega) \rho\right. \\
& \left.-\frac{1}{2} \rho A_{m}^{\dagger}(\omega) A_{m}(\omega)\right] .
\end{aligned}
$$

The Lindblad generators, $A_{m}(\omega)$, are given by,

$$
A_{m}(\omega)=\sum_{E-E^{\prime}=\hbar \omega} c_{m}^{*}(E) c_{m}\left(E^{\prime}\right)|E\rangle\left\langle E^{\prime}\right|,
$$

where the summation is over all transitions with frequency, $\omega=\left(E-E^{\prime}\right) / \hbar$, and $|E\rangle$ is the eigenstate of the Hamiltonian, $H_{0}$, of the closed system with eigenvalue, $E$. The coefficients, $c_{m}(E)$, are the expansion coefficients of the energy eigenstate in the sites basis, $|m\rangle$,

$$
|E\rangle=\sum_{m} c_{m}(E)|m\rangle
$$


The rates, $\gamma(\omega)$, are given by,

$$
\gamma(\omega)=2 \pi\left[J(\omega)\left(1+n_{T}(\omega)\right)+J(-\omega) n_{T}(-\omega)\right]
$$

where $n_{T}(\omega)$ is the bosonic distribution function at the temperature $T$,

$$
n_{T}(\omega)=\frac{1}{e^{\hbar \omega / k_{B} T}-1},
$$

and $J(\omega)$ is the Ohmic spectral density, which we choose of the form,

$$
J(\omega)= \begin{cases}0 & \text { for } \omega<0 \\ \frac{E_{R} \omega}{\hbar \omega_{c}} e^{-\omega / \omega_{c}} & \text { for } \omega>0 .\end{cases}
$$

Eq. (23) implicitly defines the reorganization energy, $E_{R}$, and the cut-off frequency, $\omega_{c}$.

Note that this form of master equation does not assume weak coupling to the sinks, only to the phonon bath. We also assumed that a strong coupling to the sinks does not influence the phonon coupling. Further work is in progress to check the validity of this assumption. 